University of Nebraska - Lincoln

DigitalCommons@University of Nebraska - Lincoln

Norman R. Simon Papers

Research Papers in Physics and Astronomy

10-1-1983

\title{
Temperature-Grid Coordinates For Treating Pulsations In The Hydrogen Ionization Zone
}

\author{
T. Aikawa \\ University of Nebraska-Lincoln \\ Norman R. Simon \\ University of Nebraska - Lincoln, nsimon@unl.edu
}

Follow this and additional works at: https://digitalcommons.unl.edu/physicssimon

Aikawa, T. and Simon, Norman R., "Temperature-Grid Coordinates For Treating Pulsations In The Hydrogen Ionization Zone" (1983). Norman R. Simon Papers. 34.

https://digitalcommons.unl.edu/physicssimon/34

This Article is brought to you for free and open access by the Research Papers in Physics and Astronomy at DigitalCommons@University of Nebraska - Lincoln. It has been accepted for inclusion in Norman R. Simon Papers by an authorized administrator of DigitalCommons@University of Nebraska - Lincoln. 
The Astrophysical Journal, 273:346-354, 1983 October 1 (C) 1983. The American Astronomical Society. All rights reserved. Printed in U.S.A.

TEMPERATURE-GRID COORDINATES FOR TREATING PULSATIONS

IN THE HYDROGEN IONIZATION ZONE

\author{
Toshiki Aikawa and Norman R. Simon \\ Department of Physics and Astronomy, University of Nebraska-Lincoln \\ Received 1982 November 15; accepted 1983 March 15
}

\begin{abstract}
A temperature grid is used for the description of motion in the hydrogen ionization region (HIR). This means zones are labeled by temperature rather than mass. Outside the HIR, the conventional Lagrangian grid is used, with transitional coordinates at the boundaries. Although our final object is to apply this scheme to the nonlinear periodic integration problem, we have first constructed a linear version as a test. It is found that periods and growth rates agree very well with those calculated using pure Lagrangian coordinates, but the temperature grid scheme proves less sensitive to a reduction of zones in the HIR. This property is desirable for the nonlinear problem where zoning must necessarily be coarse. As a further test of the temperature grid, we formulate the linear work integral in the new coordinates and confirm the consistency of the growth rates. Finally, it is shown that the scheme is insensitive to a change in the manner in which the transition zones are treated.
\end{abstract}

Subject headings: hydrodynamics - stars: interiors - stars: pulsation

\title{
I. INTRODUCTION
}

In the modeling of stellar pulsations, fully nonlinear, hydrodynamic codes are necessary, both for detailed comparisons with the observed variations of stars and for treating the difficult problem of modal selection. The approaches to hydrodynamic modeling in this field have generally been of two sorts. Beginning with the work of Christy (1966), many authors have undertaken initial value integrations by which the pulsation is built up gradually from small amplitude. In recent years this technique has been improved by the DYN code developed by Castor, Davis, and Davison (1977). As described by Davis and Davison (1978) and Davis, Moffett, and Barnes (1981), this code has been constructed with a non-Lagrangian, dynamic zoning method in the hydrogen ionization region (HIR). By use of this technique, the behavior of the pulsation in the HIR can be described in detail (Adams and Castor 1979). However, while initial value integrations can be used effectively in comparing observed light and velocity curves with their theoretical counterparts, the method becomes very inefficient in the study of modal selection.

Better suited to the modal selection problem is the second approach which involves a nonlinear periodic integration technique (Baker and von Sengbusch 1969; Stellingwerf 1974). This technique was employed by Stellingwerf $(1975 a, b)$ who tested the stability of single-mode limit cycles and proposed modal selection categories on the basis of whether or not linear perturbations corresponding to one of the modes would grow around the limit cycle of the other. Although this method is more economical than the usual initial value integrations and also allows direct computation of the stability of each mode, there are some discrepancies among the results obtained for the same models (Cox, Hodson, and Davey 1976). In at least one case, it was found that differences in modal stability could be attributed to differences in driving in the HIR (Cox 1980). Furthermore, the same study demonstrated that changes in artificial viscosity (which as a practical matter affects only the HIR) could alter modal selection properties. Given these circumstances, it seems desirable to seek an improved treatment of the HIR for use in conjunction with a strictly periodic code.

Following a suggestion by Stellingwerf (private communication), we attempt to use a temperature grid for the description of pulsations in the HIR. This means that zones are labeled by their static model temperatures rather than masses, and that we follow shells of constant temperature as the pulsation proceeds. Because the HIR boundaries are sensitive to the temperature, we can thus confine the HIR within certain zones of the temperature grid during the pulsation. To connect the temperature grid region with the ordinary Lagrangian coordinates used elsewhere in the model, transition domains must be set up at the upper and lower boundaries of the HIR.

In the DYN code, the mass rezoning in the HIR is determined essentially to satisfy the Rankine-Hugoniot jump conditions which are evaluated from the dynamical properties of two zones: one close to $8000 \mathrm{~K}$ and one close to $15,000 \mathrm{~K}$. On the other hand, in the present scheme, the mass rezoning is set up to assure that zones within the HIR retain their static model temperatures. To apply any hydrodynamic scheme to the nonlinear periodic integration technique, one must construct the Floquet matrix (e.g., Cox, Hudson, and Davey 1976) for each period. For non- 


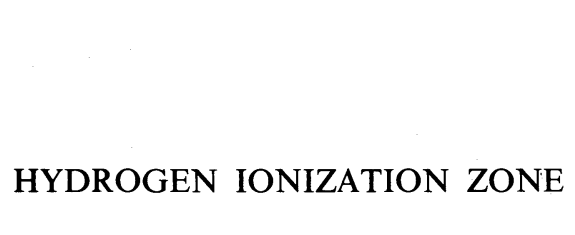

Lagrangian codes, zonal masses are not fixed but are related to other physical quantities. Thus, to calculate elements of the Floquet matrix, contributions from mass 'rézoning must be included. Because the DYN code, designed for initial value integrations, contains a complicated,algorithm for rezoning in the HIR, it is not convenient to apply this scheme directly to the strictly periodic integration technique. On the other hand, in the temperature grid system, mass motion in the HIR is determined in a relatively simple manner and thus ought to be more readily adaptable to the Baker-von Sengbusch-Stellingwerf problem.

In the present study we construct a linear version of our scheme to demonstrate its characteristics and to compare the results with those obtained using a standard Lagrangian grid. In $\S 2$, the fundamental equations are derived by using transformations from the Lagrangian grid. In $\S 3$, we formulate the eigenvalue problem in a manner similar to that of Castor (1971). In $\S 4$, we discuss the characteristics of our scheme, comparing the present results with those generated by a pure Lagrangian system.

\section{TRANSFORMATION}

We begin with the convention Lagrangian equations:

$$
\begin{gathered}
\left(\frac{\partial r}{\partial t}\right)_{M_{r}}=U \\
\left(\frac{\partial U}{\partial t}\right)_{M_{r}}=-\frac{G M_{r}}{r^{2}}-4 \pi r^{2}\left(\frac{\partial P}{\partial M_{r}}\right)_{t} \\
\left(\frac{\partial E}{\partial t}\right)_{M_{r}}+P\left(\frac{\partial V}{\partial t}\right)_{M_{r}}=-\left(\frac{\partial L_{r}}{\partial M_{r}}\right)_{t} \\
V=\frac{\partial}{\partial M_{r}}\left(\frac{4 \pi}{3} r^{3}\right)_{t} \\
L_{r}=-\frac{4\left(4 \pi r^{2}\right)^{2} \sigma}{3 \kappa}\left(\frac{\partial T^{4}}{\partial M_{r}}\right)_{r} .
\end{gathered}
$$

Following Castor, Davis, and Davison (1977), we use in the temperature grid and transition regions zonal interfaces that are fixed not in mass but in some other coordinate denoted by $x$. We employ the following identity:

$$
\left(\frac{\partial f}{\partial t}\right)_{M_{r}}=\left(\frac{\partial f}{\partial t}\right)_{x}-\left(\frac{\partial f}{\partial x}\right)_{t}\left(\frac{\partial M_{r}}{\partial x}\right)_{t}^{-1}\left(\frac{\partial M_{r}}{\partial t}\right)_{x}
$$

Here $f$ is any physical variable, and $M_{r}$, the mass within a distance $r$ of the center, is a function of $x$ and $t$.

Combining equation (6) with equations (1)-(5) gives

$$
\begin{gathered}
\left(\frac{\partial M_{r}}{\partial x}\right)_{t}\left(\frac{\partial r}{\partial t}\right)_{x}=\left(\frac{\partial M_{r}}{\partial x}\right)_{t} U+\left(\frac{\partial r}{\partial x}\right)_{t}\left(\frac{\partial M_{r}}{\partial t}\right)_{x} \\
\left(\frac{\partial M_{r}}{\partial x}\right)_{t}\left(\frac{\partial U}{\partial t}\right)_{x}=-\frac{G M_{r}}{r^{2}}\left(\frac{\partial M_{r}}{\partial x}\right)_{t}-4 \pi r^{2}\left(\frac{\partial P}{\partial x}\right)_{t}+\left(\frac{\partial U}{\partial x}\right)_{t}\left(\frac{\partial M_{r}}{\partial t}\right)_{x} \\
\left(\frac{\partial M_{r}}{\partial x}\right)_{t}\left[\left(\frac{\partial E}{\partial t}\right)_{x}+P\left(\frac{\partial V}{\partial t}\right)_{x}=-\left(\frac{\partial L_{r}}{\partial x}\right)_{t}+\left[\left(\frac{\partial E}{\partial x}\right)_{t}+P\left(\frac{\partial V}{\partial x}\right)_{t}\right]\left(\frac{\partial M_{r}}{\partial t}\right)_{x},\right. \\
\left(\frac{\partial M_{r}}{\partial x}\right)_{t} V=\frac{\partial}{\partial x}\left(\frac{4 \pi}{3} r^{3}\right)_{t} \\
\left(\frac{\partial M_{r}}{\partial x}\right)_{t} L_{r}=-\frac{4\left(4 \pi r^{2}\right)^{2} \sigma}{3 \kappa}\left(\frac{\partial T^{4}}{\partial x}\right)_{t} .
\end{gathered}
$$

Equations (7)-(11) are now converted to finite difference form. As in Castor, Davis, and Davison (1977), the spatial zoning is indicated by a subscript which is a half-odd integer for zonal quantities and an integer for interface quantities. The index increases with radius. Thus $I=1$ denotes the lower boundary, and the interface $(N+1)$ denotes the surface. Then, the equations become

$$
\begin{gathered}
D M 2_{I}\left(\frac{\partial r_{I}}{\partial t}\right)=D M 2_{I} U_{I}+\frac{1}{4}\left(r_{I+1}-r_{I-1}\right)\left(\frac{\partial M_{I-1 / 2}}{\partial t}+\frac{\partial M_{I+1 / 2}}{\partial t}\right) \\
D M 2_{I}\left(\frac{\partial U_{I}}{\partial t}\right)=-\frac{G M_{I}}{r_{I}^{2}} D M 2_{I}-4 \pi r^{2}\left(P_{I+1 / 2}-P_{I-1 / 2}\right)+\frac{1}{4}\left(U_{I+1}-U_{I-1}\right)\left(\frac{\partial M_{I-1 / 2}}{\partial t}+\frac{\partial M_{I+1 / 2}}{\partial t}\right)
\end{gathered}
$$




$$
\begin{gathered}
D M 1_{I+1 / 2}\left(\frac{\partial E_{I+1 / 2}}{\partial t}+P_{I+1 / 2} \frac{\partial V_{I+1 / 2}}{\partial t}\right)=-\left(L_{I+1}-L_{I}\right) \\
+\frac{1}{2}\left[\left(E_{I+1+1 / 2}-E_{I-1+1 / 2}\right)+P_{I+1 / 2}\left(V_{I+1+1 / 2}-V_{I-1+1 / 2}\right)\right]\left(\frac{\partial M_{I+1 / 2}}{\partial t}\right) \\
D M 1_{I+1 / 2} V_{I+1 / 2}=\frac{4 \pi}{3}\left(r_{I+1}^{3}-r_{I}^{3}\right) \\
D M 2_{I} L_{I}=\frac{4 \sigma}{3 \kappa_{I}}\left(4 \pi r_{I}^{2}\right)^{2}\left(T_{I-1 / 2}^{4}-T_{I+1 / 2}^{4}\right)
\end{gathered}
$$

where

$$
D M 1_{I+1 / 2}=M_{I+1}-M_{I}, \quad D M 2_{I}=\frac{1}{2}\left(D M 1_{I-1 / 2}+D M 1_{I+1 / 2}\right) .
$$

To perform nonlinear calculations, the time derivatives also must be converted to finite difference form.

So far, we have not specified the general coordinate, $x$. In what follows we outline a procedure whereby the temperature grid is used in the HIR, while outside of this region, the Lagrangian grid is adopted.

Putting

$$
T\left(\frac{\partial S}{\partial t}\right)_{x}=\left[\left(\frac{\partial E}{\partial t}\right)_{x}+P\left(\frac{\partial V}{\partial t}\right)_{x}\right]-\left[\left(\frac{\partial E}{\partial x}\right)_{t}+P\left(\frac{\partial V}{\partial x}\right)_{t}\right]\left(\frac{\partial M_{r}}{\partial x}\right)_{t}^{-1}\left(\frac{\partial M_{r}}{\partial t}\right),
$$

we obtain the following equation

$$
\left[\frac{1}{T} \frac{\partial T}{\partial x}+\left(\Gamma_{3}-1\right) \frac{1}{V} \frac{\partial V}{\partial x}\right]_{t}\left(\frac{\partial M_{r}}{\partial t}\right)_{x}=\left(\frac{\partial M_{r}}{\partial x}\right)_{t}\left[\frac{1}{T}\left(\frac{\partial T}{\partial t}\right)_{x}-\left(\Gamma_{3}-1\right) \frac{1}{\rho}\left(\frac{\partial \rho}{\partial t}\right)_{x}-\frac{1}{C_{v}}\left(\frac{\partial S}{\partial t}\right)_{x}\right] .
$$

Here we used a thermodynamic identity which is given in our system as

$$
\begin{aligned}
\left(\frac{\partial E}{\partial t}\right)_{x}= & T C_{v}\left[\frac{1}{T}\left(\frac{\partial T}{\partial t}\right)_{x}-\frac{1}{T}\left(\frac{\partial T}{\partial x}\right)_{t}\left(\frac{\partial M_{r}}{\partial x}\right)_{t}^{-1}\left(\frac{\partial M_{r}}{\partial t}\right)_{x}\right] \\
& +P V\left(\chi_{T}-1\right)\left[-\frac{1}{\rho}\left(\frac{\partial \rho}{\partial t}\right)_{x}-\frac{1}{V}\left(\frac{\partial V}{\partial x}\right)_{t}\left(\frac{\partial M_{r}}{\partial x}\right)^{-1}\left(\frac{\partial M_{r}}{\partial t}\right)_{x}\right]+\left(\frac{\partial E}{\partial x}\right)_{t}\left(\frac{\partial M_{r}}{\partial x}\right)^{-1}\left(\frac{\partial M_{r}}{\partial t}\right)_{x} .
\end{aligned}
$$

Converting to difference form and using the time derivative of equation (10):

$$
\begin{aligned}
H B 1_{I, 1} \frac{\partial M_{I-1+1 / 2}}{\partial t} & +H B 1_{I, 2} \frac{\partial M_{I+1 / 2}}{\partial t}+H B 1_{I, 3} \frac{\partial M_{I+1+1 / 2}}{\partial t} \\
= & D M 1_{I+1 / 2}\left[\frac{1}{T}\left(\frac{\partial T}{\partial t}\right)_{I+1 / 2}+\frac{4 \pi\left(\Gamma_{3}-1\right)_{I+1 / 2}}{V_{I+1 / 2} D M 1_{I+1 / 2}}\left(r_{I+1}^{2} \frac{\partial r_{I+1}}{\partial t}-r_{I}^{2} \frac{\partial r_{I}}{\partial t}\right)-\frac{1}{\left(C_{v}\right)_{I+1 / 2}}\left(\frac{\partial S}{\partial t}\right)_{I+1 / 2}\right],
\end{aligned}
$$

where

$$
\begin{aligned}
& H B 1_{I, 1}=\frac{1}{2}\left(\Gamma_{3}-1\right)_{I+1 / 2}, \\
& H B 1_{I, 2}=\frac{1}{2}\left[\frac{T_{I+1+1 / 2}-T_{I-1+1 / 2}}{T_{I+1 / 2}}+\left(\Gamma_{3}-1\right)_{I+1 / 2} \frac{V_{I+1+1 / 2}-V_{I-1+1 / 2}}{V_{I+1 / 2}}\right], \\
& H B 1_{I, 3}=-\frac{1}{2}\left(\Gamma_{3}-1\right)_{I+1 / 2} .
\end{aligned}
$$

This equation determines the mass change of the $\left(I+\frac{1}{2}\right)$ shell in terms of changes in the temperature, the radius, and the entropy. In the HIR, whose zonal boundaries are indicated as $I \in\left[I_{A F}, I_{B F}\right]$, we use equation (20) with $(\partial T / \partial t)=0$ for determination of the mass flow. On the other hand, above and below the HIR, where special techniques are not needed, it is simpler and more efficient to employ the usual Lagrangian grid $(\partial M / \partial t)=0$. We thus have the problem of connecting the regions of different coordinates, and for this purpose use the following equation instead of equation (20):

$$
\begin{aligned}
& F 1_{I} \cdot H B 1_{I, 1} \frac{\partial M_{I-1+1 / 2}}{\partial t}+H B 1_{I, 2} \frac{\partial M_{I+1 / 2}}{\partial t}+F 1_{I} \cdot H B 1_{I, 3} \frac{\partial M_{I+1+1 / 2}}{\partial t} \\
&=F 2_{I} \cdot D M 1_{I+1 / 2}\left[\frac{4 \pi\left(\Gamma_{3}-1\right)_{I+1 / 2}}{V_{I+1 / 2} D M 1_{I+1 / 2}}\left(r_{I+1}^{2} \frac{\partial r_{I+1}}{\partial t}-r_{I}^{2} \frac{\partial r_{I}}{\partial t}\right)-\frac{1}{\left(C_{v}\right)_{I+1 / 2}}\left(\frac{\partial S}{\partial t}\right)_{I+1 / 2}\right] .
\end{aligned}
$$


Here $F 1_{I}$ and $F 2_{I}$ are weight functions by which the different' regions are connected smoothly. For $I \in\left[I_{A F}, I_{B F}\right]$, we set $F 1_{I}=F 2_{I}=1$ in equation (22). This means that the itemperature grid is used in this region. To set up transition domains both below and above the temperature 'grid region, we use a smooth function which changes from zero to 1 for $I \in\left[I_{A}, I_{A F}\right]$ and from 1 to zero for $I \in\left[I_{B F}, I_{B}\right]$. Thus we have Lagrangian coordinates for zone numbers $I<I_{A}$ and $I>I_{B}$, and the temperature grid for $I \in\left[I_{A F}, I_{B F}\right]$. In the transition domains $\left(I \in\left[I_{A}, I_{A F}\right], I \in\left[I_{B F}, I_{B}\right]\right)$, both mass and temperature are allowed to vary. Consistent with these boundaries we now slightly redefine the HIR to consist of $I \in\left[I_{A}, I_{B}\right]$.

\section{EIGENVALUE PROBLEM}

To make sure our scheme works well, we apply it to the linear nonadiabatic eigenvalue problem and compare our results with those obtained with a pure Lagrangian scheme. We note that in general the present technique allows simultaneous perturbation of the static values of both mass and temperature.

To begin, we linearize equations (12)-(16) and equation (22). The linearly perturbed quantities are indicated by the prefix $\delta$. We replace $\delta r_{I}, \delta S_{I+1 / 2}$, and $\delta M_{I+1 / 2}$ with $X_{I}, Y_{I}$, and $Z_{I}$ defined as follows:

$$
\begin{aligned}
X_{I} & =\left(D M 2_{I}\right)^{1 / 2}\left[\delta r_{I}-\frac{1}{4}\left(\frac{r_{I+1}-r_{I-1}}{D M 2_{I}}\right)\left(\delta M_{I-1 / 2}+\delta M_{I+1 / 2}\right)\right], \\
Y_{I} & =T_{I+1 / 2} \delta S_{I+1 / 2} \\
Z_{I} & =\delta M_{I+1 / 2} .
\end{aligned}
$$

Then, in terms of these variables, we get from equation (15)

$$
\left(\frac{\delta \rho}{\rho}\right)_{I+1 / 2}=D R 1_{I, 1} X_{I}+D R 1_{I, 2} X_{I+1}+D R 2_{I, 1} Z_{I-1}+D R 2_{I, 2} Z_{I}+D R 2_{I, 3} Z_{I+1}
$$

where $D R 1_{I, 1}$ and $D R 1_{I, 2}$ are just $D R_{I, 1}$ and $D R_{I, 2}$ as defined by Castor (1971), and $D R 2_{I, 1}, D R 2_{I, 2}$, and $D R 2_{I, 3}$ are given by

$$
\begin{aligned}
& D R 2_{I, 1}=\frac{1}{D M 1_{I+1 / 2} V_{I+1 / 2}}\left[\frac{4 \pi r_{I}^{2}\left(r_{I+1}-r_{I-1}\right)}{4 D M 2_{I}}-\frac{1}{2} V_{I+1 / 2}\right], \\
& D R 2_{I, 2}=\frac{4 \pi}{4 D M 1_{I+1 / 2} V_{I+1 / 2}}\left[\frac{r_{I}^{2}\left(r_{I+1}-r_{I-1}\right)}{D M 2_{I}}-\frac{r_{I+1}^{2}\left(r_{I+2}-r_{I}\right)}{D M 2_{I+1}}\right], \\
& D R 2_{I, 3}=\frac{1}{D M 1_{I+1 / 2} V_{I+1 / 2}}\left[\frac{1}{2} V_{I+1 / 2}-\frac{4 \pi r_{I+1}^{2}\left(r_{I+2}-r_{I}\right)}{4 D M 2_{I+1}}\right] .
\end{aligned}
$$

From thermodynamic identities, we obtain the perturbations for $T$ and $P$ :

$$
\begin{aligned}
\left(\frac{\delta T}{T}\right)_{I+1 / 2}= & \left(\Gamma_{3}-1\right)_{I+1 / 2}\left(D R 1_{I, 1} X_{I}+D R 1_{I, 2} X_{I+1}+D R 2_{I, 1} Z_{I-1}+D R 2_{I, 2} Z_{I}\right. \\
& \left.+D R 2_{I, 3} Z_{I+1}\right)+E X 2_{I+1 / 2} Z_{I}+\left[\frac{\rho\left(\Gamma_{3}-1\right)}{P}\right]_{I+1 / 2} Y_{I} \\
\left(\frac{\delta P}{P}\right)_{I+1 / 2}= & \left(\Gamma_{1}\right)_{I+1 / 2}\left(D R 1_{I, 1} X_{I}+D R 1_{I, 2} X_{I+1}+D R 2_{I, 1} Z_{I-1}+D R 2_{I, 2} Z_{I}\right. \\
& \left.+D R 2_{I, 3} Z_{I+1}\right)+\left(\chi_{T}\right)_{I+1 / 2} E X 2_{I+1 / 2} Z_{I}+\left(\frac{\chi_{T}}{C_{v} T}\right)_{I+1 / 2} Y_{I} .
\end{aligned}
$$

where $\left(\Gamma_{3}-1\right)$ and $\Gamma_{1}$ have the usual meaning, $\chi_{T}=(\partial \ln P / \partial \ln T)_{\rho}$, and $E X 2$ is defined as:

$$
E X 2_{I+1 / 2}=\left[\frac{1}{T} \frac{d T}{d M}+\left(\Gamma_{3}-1\right) \frac{1}{V} \frac{d V}{d M}\right]=\frac{d V}{d M} \cdot\left[\frac{d \ln T}{d \ln V}-\left(\frac{d \ln T}{d \ln V}\right)_{a d}\right] .
$$

The linearization of equation (16) gives the following equation:

$$
\begin{aligned}
E X 2_{I+1 / 2}= & \frac{1}{2}\left[\frac{1}{T_{I+1 / 2}}\left(\frac{T_{I+1+1 / 2}-T_{I+1 / 2}}{D M 2_{I+1}}+\frac{T_{I+1 / 2}-T_{I-1+1 / 2}}{D M 2_{I}}\right)\right. \\
& \left.+\frac{\left(P_{3}-1\right)_{I+1 / 2}}{V_{I+1 / 2}}\left(\frac{V_{I+1+1 / 2}-V_{I+1 / 2}}{D M 2_{I+1}}+\frac{V_{I+1 / 2}-V_{I-1+1 / 2}}{D M 2_{I}}\right)\right] .
\end{aligned}
$$


where the $B L 1$ and $B L 2$ values are just the same as defined by Castor (1971) except that we use the $T^{4}$ harmonic mean between $\kappa_{I-1 / 2}$ and $\kappa_{I+1 / 2}$ to calculate the shell opacity i $\kappa_{I}$ (Christy 1964; Stobie 1969).

To search for normal modes, we assume that the time dependence is exponential. Using the linearized equations (12), (13), and (14), we obtain finally

$$
\begin{aligned}
\omega^{2} X_{I}= & G 1_{I, 1} X_{I-1}+G 1_{I, 2} X_{I}+G 1_{I, 3} X_{I+1}+G 2_{I, 1} Y_{I-1}+G 2_{I, 2} Y_{I} \\
& +G 3_{I, 1} Z_{I-2}+G 3_{I, 2} Z_{I-1}+G 3_{I, 3} Z_{I}+G 3_{I, 4} Z_{I+1} \\
i \omega Y_{I}= & K 1_{I, 1} X_{I-1}+K 1_{I, 2} X_{I}+K 1_{I, 3} X_{I+1}+K 1_{I, 4} X_{I+2} \\
& +K 2_{I, 1} Y_{I-1}+K 2_{I, 2} Y_{I}+K 2_{I, 3} Y_{I+1} \\
& +K 3_{I, 1} Z_{I-2}+K 3_{I, 2} Z_{I-1}+K 3_{I, 3} Z_{I}+K 3_{I, 4} Z_{I+1}+K 3_{I, 5} Z_{I+2} .
\end{aligned}
$$

Here $\omega$ is the complex eigenfrequency. Similarly equation (12) is converted to

$$
H 1_{I, 1} X_{I}+H 1_{I, 2} X_{I+1}+H 2_{I, 1} Y_{I}+H 3_{I, 1} Z_{I-1}+H 3_{I, 2} Z_{I}+H 3_{I, 3} Z_{I+1}=0 .
$$

Outside the HIR, $Z_{I}$ will be zero, and so we can solve equations (34) and (35) with the same boundary conditions used in a pure Lagrangian scheme. To solve equation (36) for $Z_{I}$, we once more set $Z_{I}=0$ outside the HIR. In practice, we solve alternately the band matrix of equations (34) and (35) for $X_{I}$ and $Y_{I}$, and the tridiagonal matrix of equation (36) for $Z_{I}$, beginning with equations (34) and (35) in the HIR with $Z_{I}=0$ as a first guess. About 10 iterations are needed to get four-figure accuracy for the growth rates.

\section{RESULTS AND DISCUSSION}

We apply equations (34)-(36) to the models designated by numbers $3,7,10$, and 13 in Baker and Kippenhahn (1965). Convection is completely neglected in our analysis. The Los Alamos opacities are used in the form of Stellingwerf's $(1975 a, b)$ analytical expression. The mass chosen for all models is $7 M_{\odot}$, and the chemical composition is $X=0.60, Y=0.36, Z=0.04$. The envelopes extend from a surface with optical depth $\tau=0.001$ to a lower boundary which is just less than 0.10 of the stellar radius.

The models are constructed in such a way that the HIR is finely zoned. Figure 1 displays the typical zoning and the HIR. We use the temperature grid for those zones whose temperature satisfies the relation $0.7 \leq T_{I+1 / 2} \leq 1.7$ in units of $10^{4} \mathrm{~K}$. On both sides of this region, lie the transition domains which are characterized by $0.6 \leq T_{I+1 / 2} \leq 0.7$ and $1.7 \leq T_{I+1 / 2} \leq 1.8$, respectively. Thus, the HIR in this case is defined as consisting of those zones whose static temperature satisfies $0.6 \leq T_{I+1 / 2} \leq 1.8$.

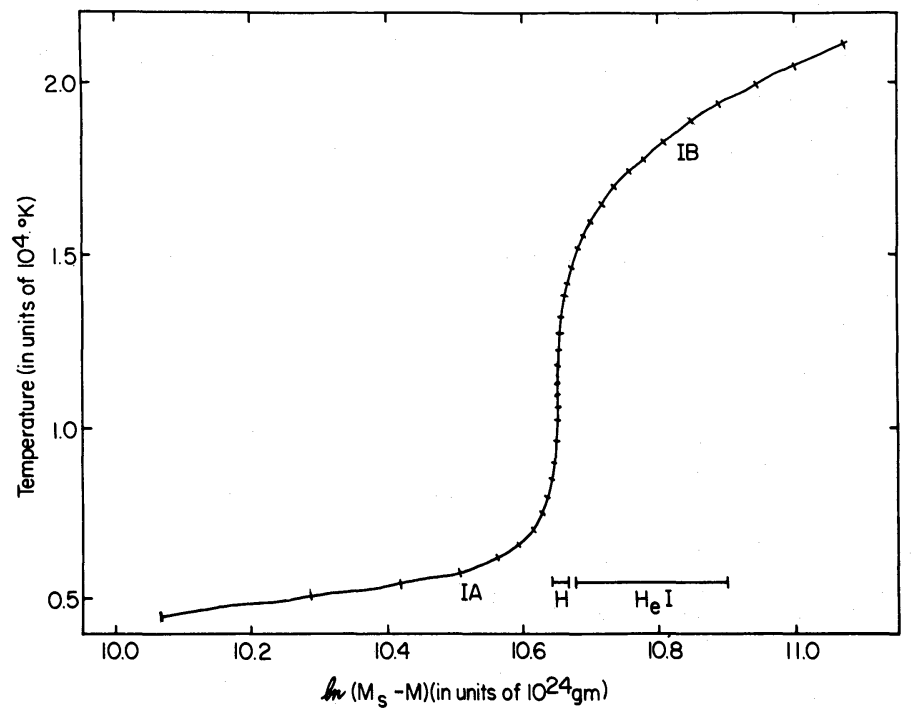

FIG. 1.-Typical zoning for the HIR and surrounding regions. $M_{S}$ is the total mass of the star. The marks indicate zonal boundaries. $I_{A}$ and $I_{B}$ give the boundaries of the HIR. The actual $\mathrm{H} \mathrm{I}$ and $\mathrm{He} \mathrm{I}$ ionization zones are shown at the bottom. 
TABLE', 1 '

Zoning Characteristícs (Model 7)

\begin{tabular}{cccc}
\hline \hline Type & $\begin{array}{c}\text { Total Number } \\
\text { of Zones }\end{array}$ & $\begin{array}{c}\text { I Number of Zones } \\
\text { in HIR }\end{array}$ & $\Delta T(\%)$ \\
\hline$C \ldots \ldots \ldots \ldots \ldots \ldots$ & 57 & 13 & 14 \\
$S \ldots \ldots \ldots \ldots \ldots \ldots$ & 112 & 25 & 6 \\
$F \ldots \ldots \ldots \ldots \ldots \ldots$ & 183 & 41 & 4 \\
$F F \ldots \ldots \ldots \ldots \ldots$ & 247 & 55 & 2 \\
\hline
\end{tabular}

The weight functions $F 1_{I}$ and $F 2_{I}$ used here are as follows:

$$
\begin{array}{ll}
F 1_{I}=F 2_{I}=1.0 \exp \left(-2 \frac{T_{I+1 / 2}-T_{I A+1 / 2}}{T_{I A+1 / 2}-T_{I F A+1 / 2}}\right) & \left(\text { for } I \in\left[I_{A}, I_{F A}\right]\right), \\
F 1_{I}=F 2_{I}=1.0 & \left(\text { for } I \in\left[I_{F A}, I_{F B}\right]\right), \\
F 1_{I}=F 2_{I}=1.0 \exp \left(-2 \frac{T_{I B+1 / 2}-T_{I+1 / 2}}{T_{I B+1 / 2}-T_{I F B+1 / 2}}\right) & \left(\text { for } I \in\left[I_{F B}, I_{B}\right]\right) .
\end{array}
$$

Although $F 1_{I}$ and $F 2_{I}$ are independent weight functions, we put $F 1_{I}=F 2_{I}$ for simplicity in the linear version.

To check the effects of fine zoning on the calculated results, four kinds of zoning were tested for the same model. The zoning characteristics are summarized in Table 1. The zoning becomes finer along the sequence: $C, S, F, F F$. The last column expresses the temperature difference from one shell to the next in the HIR. The zoning types $S$ and $C$ would be typical for a linear nonadiabatic analysis and a nonlinear hydrodynamic calculation respectively.

The fundamental period $P_{0}$ and the period ratios and growth rates up to the fourth overtone are summarized in Table 2 for the Baker and Kippenhahn (1965) models with $S$ type zoning. The growth rate $\eta$ is defined as $-4 \pi \omega_{i} / \omega_{r}$, where $\omega_{r}$ and $\omega_{i}$ are the real and imaginary parts of the complex frequency $\omega$. For comparison, Table 2 presents the corresponding results obtained with the pure Lagrangian grid, designated by $L$. Although there are slight differences in some of the entries, the overall agreement is very good.

In Table 3 we display the same quantities for $C$ type models. One notices the tendency for the coarsely zoned models to have slightly larger periods as well as less driving (or greater dissipation). The latter effect is much more

TABLE 2

BaKer AND KipPenhahn Series ( $S$ Models)

\begin{tabular}{ccccccccccc}
\hline \hline Model & \multicolumn{1}{c}{$P_{0}$} & $\eta_{0}$ & $P_{1} / P_{0}$ & $\eta_{1}$ & $P_{2} / P_{0}$ & $\eta_{2}$ & $P_{3} / P_{0}$ & $\eta_{3}$ & $P_{4} / P_{0}$ & $\eta_{4}$ \\
\hline $3 \ldots \ldots \ldots \ldots \ldots$ & 16.231 & +5.8 & 0.6990 & +3.7 & 0.5141 & -8.2 & 0.4031 & -16.0 & 0.3299 & -17.4 \\
$3 L \ldots \ldots \ldots \ldots \ldots$ & 16.233 & +5.7 & 0.6992 & +3.3 & 0.5143 & -8.9 & 0.4033 & -17.0 & 0.3301 & -18.3 \\
$7 \ldots \ldots \ldots \ldots \ldots$ & 11.345 & +3.2 & 0.7100 & +6.6 & 0.5397 & -4.3 & 0.4286 & -18.2 & 0.3530 & -24.2 \\
$7 L \ldots \ldots \ldots \ldots \ldots$ & 11.346 & +3.1 & 0.7102 & +6.3 & 0.5400 & -4.6 & 0.4290 & -18.6 & 0.3533 & -24.4 \\
$10 \ldots \ldots \ldots \ldots \ldots$. & 8.954 & +0.8 & 0.7145 & +4.6 & 0.5538 & -2.1 & 0.4440 & -17.7 & 0.3686 & -27.3 \\
$10 L \ldots \ldots \ldots \ldots \ldots$ & 8.955 & +0.8 & 0.7147 & +4.4 & 0.5541 & -2.4 & 0.4443 & -17.9 & 0.3688 & -27.1 \\
$13 \ldots \ldots \ldots \ldots \ldots$ & 7.207 & -1.2 & 0.7186 & +0.3 & 0.5649 & -2.6 & 0.4575 & -17.2 & 0.3832 & -28.7 \\
$13 L \ldots \ldots \ldots \ldots$ & 7.208 & -1.2 & 0.7188 & +0.0 & 0.5651 & -2.9 & 0.4577 & -17.2 & 0.3835 & -28.2 \\
\hline
\end{tabular}

NoTE. $-P_{0}$ is given in units of days; $\eta$, in units of $10^{-2}$.

TABLE 3

Baker and Kippenhahn Series ( $C$ Models)

\begin{tabular}{crrrrrrrrrr}
\hline \hline Model & \multicolumn{1}{c}{$P_{0}$} & $\eta_{0}$ & $P_{1} / P_{0}$ & $\eta_{1}$ & $P_{2} / P_{0}$ & $\eta_{2}$ & $P_{3} / P_{0}$ & $\eta_{3}$ & $P_{4} / P_{0}$ & $\eta_{4}$ \\
\hline $3 \ldots \ldots \ldots \ldots \ldots$ & 16.320 & +5.4 & 0.6993 & +3.7 & 0.5172 & -7.6 & 0.4060 & -15.8 & 0.3332 & -17.8 \\
$3 L \ldots \ldots \ldots \ldots$. & 16.328 & +4.9 & 0.7002 & +1.4 & 0.5181 & -10.4 & 0.4070 & -19.4 & 0.3341 & -21.2 \\
$7 \ldots \ldots \ldots \ldots \ldots$ & 11.412 & +2.8 & 0.7099 & +5.8 & 0.5418 & -4.4 & 0.4308 & -18.3 & 0.3555 & -24.8 \\
$7 L \ldots \ldots \ldots \ldots$. & 11.416 & +2.4 & 0.7107 & +4.7 & 0.5429 & -5.7 & 0.4301 & -19.5 & 0.3568 & -25.0 \\
$10 \ldots \ldots \ldots \ldots$ & 9.013 & +0.5 & 0.7149 & +3.5 & 0.5560 & -2.5 & 0.4464 & -17.5 & 0.3716 & -27.3 \\
$10 L \ldots \ldots \ldots \ldots$ & 9.016 & +0.3 & 0.7155 & +2.8 & 0.5569 & -3.5 & 0.4476 & -17.8 & 0.3726 & -26.1 \\
$13 \ldots \ldots \ldots \ldots$ & 7.255 & -1.3 & 0.7192 & -0.4 & 0.5666 & -3.1 & 0.4594 & -16.7 & 0.3858 & -28.4 \\
$13 L \ldots \ldots \ldots \ldots$ & 7.257 & -1.4 & 0.7197 & -1.0 & 0.5676 & -3.9 & 0.4605 & -16.6 & 0.3867 & -26.4 \\
\hline
\end{tabular}

NOTE. $-P_{0}$ is given in units of days; $\eta$, in units of $10^{-2}$. 
pronounced in the Lagrangian scheme than it is when the temperature grid is used. Figure 2 shows this situation in more detail. The growth rates obtained in both schemes are plotted against the degree of fineness of the zoning. Although the growth rates agree rather closely in the $F F$ cłse, the discrepancy between two results becomes large as the zoning grows coarser. This tendency is especially noticeable for the case of $C$ type zoning. It is clear from Figure 2 that, for every case displayed, the coarsely zoned temperature grid provides a better estimate of the finely zoned growth rate than does the coarsely zoned pure Lagrangian scheme.

To further investigate this question, we construct the pulsational work integral for the temperature grid scheme. We begin by writing the integral in fully nonlinear form (Cox and Giuli 1968):

$$
\left\langle\frac{d W}{d t}\right\rangle=\frac{1}{\Pi} \int_{0}^{\Pi} d t \int P \frac{\partial}{\partial t}\left(\frac{1}{\rho}\right)_{M_{r}} d M_{r} .
$$

Transforming to our general coordinate system, we have

$$
\begin{aligned}
\left\langle\frac{d W}{d t}\right\rangle & =\frac{1}{\Pi} \int_{0}^{\Pi} d t \int_{x} P V\left[-\frac{1}{\rho}\left(\frac{\partial \rho}{\partial t}\right)_{x}-\left(\frac{\partial \ln V}{\partial x}\right)_{t}\left(\frac{\partial M_{r}}{\partial x}\right)^{-1}\left(\frac{\partial M_{r}}{\partial t}\right)_{x}\right]\left(\frac{\partial M_{r}}{\partial x}\right)_{t} d x \\
& =-\frac{1}{\Pi} \int_{0}^{\Pi} d t\left[\int_{x} P V\left(\frac{1}{\rho} \frac{\partial P}{\partial t}\right)\left(\frac{\partial M_{r}}{\partial x}\right) d x+\int_{x} P V \frac{\partial \ln V}{\partial x}\left(\frac{\partial M_{r}}{\partial t}\right) d x\right] .
\end{aligned}
$$

To evaluate $\langle d W / d t\rangle$ in the linear theory, $P, \rho$, and $M_{r}$ are expanded to first order in the perturbations and terms up to second order are retained in the integrand. We find

$$
\begin{aligned}
\left\langle\frac{d W}{d t}\right\rangle= & -\frac{1}{\Pi} \int_{0}^{\Pi} d t\left[\int_{x} P V\left(\frac{\partial P}{P}\right) \frac{\partial}{\partial t}\left(\frac{\delta \rho}{\rho}\right) \frac{\partial M_{r}}{\partial x} d x\right. \\
& \left.+\int_{x} P V \frac{d \ln V}{d x}\left(\frac{\delta P}{P}\right)\left(\frac{\partial \delta M_{r}}{\partial t}\right) d x+\int_{x} P V \frac{d \ln P}{d x}\left(\frac{\delta \rho}{\rho}\right)\left(\frac{\partial \delta M_{r}}{\partial t}\right) d x\right] .
\end{aligned}
$$
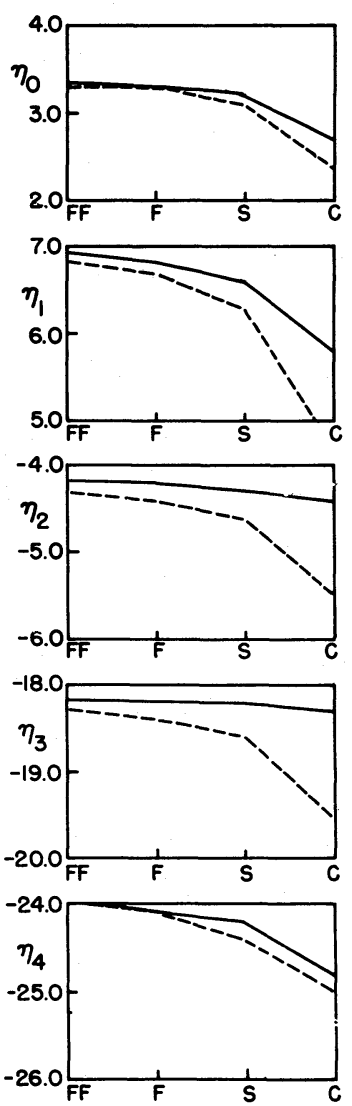

FIG. 2.-Dependence of the growth rate (in units of $10^{-2}$ ) on the fineness of zoning. The symbols for the degree of fineness are the same as in Table 1. Solid lines, temperature grid; dashed lines, pure Lagrangian grid. 
The ratio of the imaginary part to the real part of the eigenfrequency should be related to the pulsational work integral in the following way (Castor 1971):

$$
\left\langle\frac{d W}{d t}\right\rangle / K \approx=\frac{4 \pi \omega_{i}}{\omega_{r}}
$$

where $K$ is the pulsation energy, which for the present scheme is given by:

$$
K=\frac{1}{2} \int_{M_{r}}\left(\frac{\partial r}{\partial t}\right)_{M_{r}}^{2} d M_{r}=\frac{1}{2} \int_{x}\left[\left(\frac{\partial r}{\partial t}\right)_{x}-\left(\frac{\partial \ln r}{\partial x}\right)_{t}\left(\frac{\partial M_{r}}{\partial x}\right)_{t}^{-1}\left(\frac{\partial M_{r}}{\partial t}\right)\right]^{2}\left(\frac{\partial M_{r}}{\partial x}\right)_{t} d x .
$$

Using equations (40) and (42), we confirm equation (41) for our models, thus demonstrating the self-consistency of the present results.

Moreover, by evaluating the integrand of equation (40) (that is, the differential work), we can investigate the seat in the models of the difference in the growth rates between the temperature grid and pure Lagrangian formulations. Figure 3 shows the difference in the differential work $\Delta W_{I}$ (temperature grid minus pure Lagrangian) normalized by $K$ versus zone number for the first overtone of model $7 C$. As one might expect, the major difference comes from the HIR and some adjacent zones.

As an additional test of our scheme, we have solved the eigenvalue problem by using another set of weight functions in place of those given by equation (37). In particular, the temperature grid region and the Lagrangian grid region are connected by linear (rather than exponential) weight functions in the transition zones. We find that the change in weighting affects our models negligibly. Thus the temperature grid formulation does not seem sensitive to the precise manner of treating the transition domains.

Finally, we have tested the effects of the width of the temperature grid region on the eigenvalues. We find that changes in the lower boundary of the HIR between 1.6 and 2.0 in $T_{I+1 / 2}$ yield negligible changes in the eigenvalues. On the other hand, the tridiagonal matrix in equation (36) cannot be properly solved when the inner boundary of the HIR is set much lower than $T_{I+1 / 2}=2.0$. At the moment, the origin of this problem remains unclear.

\section{CONCLUSION}

Our linear tests of the temperature grid indicate that it performs well in calculating periods and growth rates and is less sensitive to coarse zoning than is a pure Lagrangian scheme. This property should be advantageous in nonlinear calculations and particularly in the modal selection problem where small differences in growth or switching rates could have important effects on modal stability. In future work we plan to study these questions in detail by constructing a temperature grid system designed for use in nonlinear, strictly periodic integrations.

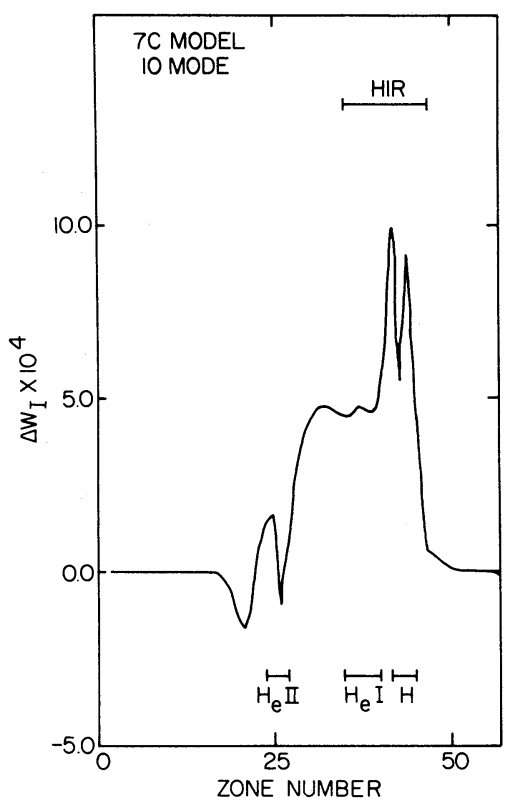

FIG. 3.-Difference in the differential work between the temperature grid and the pure Lagrangian. The extent of the HIR is indicated by a bar. 


$$
\text { i }
$$

We are grateful to R. F. Stellingwerf for suggesting the temperature grid approach and for helpful conversations. It is a pleasure to acknowledge support for this work from the National Science Foundation under grant AST 81-05064.

$\begin{array}{ll}1 & 1 \\ 1 & 1\end{array}$

\section{REFERENCES}

Adams, T. F., and Castor, J. I. 1979, Ap. J., 230, 826.

Baker, N. H., and Kippenhahn, R. 1965, Ap. J., 142, 868.

Baker, N. H., and von Sengbusch, K. 1969, Mitt. Astr. Ges., No. 27. Castor, J. I. 1971, Ap. J., 166, 109.

Castor, J. I., Davis, C. G., and Davison, D. K. 1977, Los Alamos Scientific Laboratory Report, LA-6664.

Christy, R. F. 1964, Rev. Mod. Phys., 36, 555. 1966, Ap. J., 144, 108.

Cox, A. N. 1980, Space Sci. Rev., 27, 475.

Cox, A. N., Hodson, S. W., and Davey, W. R. 1976, in Proceedings of the Solar and Stellar Pulsation Conference, ed. A. N. Cox and R. G. Deupree (LASL: LA-6544c), p. 188.
Cox, J. P., and Giuli, R. T. 1968, Principles of Stellar Structure (New York: Gordon \& Breach), Vol. 2.

Davis, C. G., and Davison, D. K. 1978, Ap. J., 221, 929.

Davis, C. G., Moffett, T. J., and Barnes, T. G. 1981, Ap. J., 246, 914. Stellingwerf, R. F. 1974, Ap. J., 192, 139. . $1975 a$, Ap. J., 195, 441. 1975b, Ap. J., 199, 705

Stobie, R. S. 1969, M.N.R.A.S., 144, 461.

Toshiki Aikawa and Norman R. Simon: Department of Physics and Astronomy, University of Nebraska-Lincoln, Lincoln, NE 68588-0111 